\title{
ELIMINATING TUBERCULOSIS: WHERE IS THE STRATEGY?
}

$\mathbf{N}$ ew South Wales must produce a strategy for the control of tuberculosis (TB) in the State. Investigations of outbreaks in NSW do not follow any written protocol and are inadequately documented. An elimination strategy would focus control and prevention measures. It should address TB surveillance, screening, prophylaxis, case follow-up and evaluation.

Recent changes in tuberculosis surveillance in NSW include the development of a database for TB notifications. This has now been incorporated into the Infectious Diseases Surveillance System - IDSS.

Other developments toward a TB strategy are:

- formation of the Tuberculosis Advisory Committee, with members representing the NSW Health Department, the public health network, the Royal Australian College of Physicians, the microbiology laboratories and the Community Health and AntiTuberculosis Association; surveillance of tuberculosis in high risk groups; population-based prevalence studies;

a review of the refugee and migrant screening program;

- rewriting all Departmental circulars related to tuberculosis; and

a a review of clinical services for tuberculosis.

The incidence of TB in developed countries has fallen dramatically over the past 150 years but, at least in NSW, this decline levelled out during the 1970 s. Incidence rates fell only slightly in the 1980 s; by 1988 the rate of TB was 6.2 per 100,000 people - lower than rates in the United States with 9 per $100,000^{1,2}$ and in the United Kingdom with 12.5 per 100,000 in 1984 .

In contrast to the situation 20 years ago, most TB cases in NSW now occur among people born outside Australia. Since the 1970s the incidence of TB among Australian-born people has continued to decline, while the incidence of TB among foreign-born people has not. Present trends suggest that with current practices TB could be eliminated from the Australian-born population within 10 years, but not from people born outside Australia now living in NSW.

About 10 per cent of people infected with Mycobacterium tuberculosis develop active TB. The risk of developing disease is highest soon after infection. Children are most likely to develop rapidly progressive disease. Age, sex, past $\mathrm{TB}$, silicosis, human immunodeficiency virus (HIV) infection and a range of other medical conditions including cancers and renal failure alter an individual's susceptibility to developing clinical disease $\mathrm{e}^{3,4}$.

There are insufficient data available on people infected with Mycobacterium tuberculosis in NSW. Such information is important for predicting the course of TB and for guiding screening procedures. All people migrating to Australia require a chest $\mathrm{x}$-ray before immigration to exclude active TB. Apart from refugees and people immigrating directly from South East Asia and Latin America, migrants to NSW are not routinely screened for TB infection. In contrast, people migrating from high prevalence countries to the UK and the US are tuberculin tested.

In NSW, TB control is the responsibility of the Health Department. Forty hospital-based chest clinic sisters are engaged in the surveillance and control of TB through therapy supervision, the investigation of case contacts and by coordinating radiological screening of migrants.

The NHMRC has produced guidelines on TB management, but not a strategy for the elimination of TB. Some States, notably South Australia, Western Australia, Queensland and the Northern Territory, are developing strategies or protocols to control TB.

In 1988 the US Advisory Committee on Immunisation Practices recommended four strategies for the control of $\mathrm{TB}^{1}$ : - early identification and treatment of people with
infectious TB;

a identification and treatment of people with non contagious TB;

- use of ventilation and ultraviolet lights to decontaminate infectious air in places where the risk of infection is exceptionally high; and

- BCG vaccination for uninfected children who are placed at unavoidable risk of TB for whom other methods of prevention and control are not feasible.

In 1989 the US Public Health Service produced a strategic plan for the elimination of TB by the year $2010^{6}$.

Elimination is defined as less than one case per million.

The three steps toward elimination outlined included:

- more effective use of existing prevention and control methods:

development and evaluation of new technologies for treatment, diagnosis and prevention; and

rapid assessment and transfer of newly developed technologies into public health practice.

Based on a review of current practices and recommendations of TB authorities in Australia and the US, we suggest the following strategy for the elimination of TB from NSW by the year 2001.

\section{SURVEILLANCE}

- Medical practitioners and laboratories should report provisional diagnosis of TB by telephone to the local Area/Regional Medical Officer of Health or the Director of the Public Health Unit (PHU) and follow up with a written notification.

PHUs should maintain a local database for TB cases using a format developed by the Epidemiology and Health Services Evaluation Branch (EHSEB).

The EHSEB should collate Statewide data, and in turn forward de-identified data to the Communicable Diseases Network Australia New Zealand.

\section{SCREENING}

- Screening people for TB infection should involve a tuberculin test. Where a person has a positive tuberculin test, or any signs or symptoms suggestive of illness, a chest x-ray should be performed to exclude active disease.

- Chest X-rays should be used to detect people with active TB. Close case contacts and homeless people over 16 years of age should have a chest $\mathrm{x}$-ray.

To identify people who could benefit from chemoprophylaxis, the following people should be screened for TB:

- contacts of people known or suspected to have clinical TB;

- immigrants from high prevalence TB countries;

- people with medical risk factors for TB;

- people from the groups found to have an annual incidence of TB infection higher than that of the general community; and

- people with HIV infection. 


\section{Eliminating tuberculosis}

- Continued from page 39

- Contacts should be screened according to risk group. Screening should be guided by the rate of infection in each group of contacts. Contacts of infectious cases should be divided into high, moderate and low risk groups. High risk would include household contacts and close associates. Moderate risk contacts may include close relatives, friends and school class or work colleagues. Low risk groups may include people from other classes or other work areas.

- In contact tracing, the high risk group should be screened with tuberculin skin tests first. If the incidence of infection in this group is greater than that of the general population (until better data are available, 5 per cent in adults and 1 per cent in children), screening should progress to the moderate risk group. Again if the incidence is greater than the general population, screening should progress to the low risk group until all those at risk are tested. Screening may stop when the incidence of infection in the risk group screened is that of the general population.

- Contacts with negative reactions should have repeat tuberculin tests after eight weeks. High risk contacts should also be retested within 12 months.

To determine community TB infection rates, the EHSEB should collate existing sources of data on TB infection among police cadets, medical students, nursing and other students.

- Chest clinics should work with PHUs to collect and collate infection and disease data arising from contact tracing. These data should be recorded on a database related to the TB database. Reports on major investigations of TB should be made available to the EHSEB within one month of identification of the index case.

The NSW Health Department should undertake tuberculin surveys of subgroups at risk of infection, such as samples of residents of long-term care institutions (including prisons and nursing homes), Aborigines and intravenous drug users, as well as school children.

To assess the value of migrant and refugee chest $\mathrm{x}$-ray screening, the EHSEB should arrange for the collection and analysis of data on TB cases diagnosed by these programs.

\section{BCG VACCINATION}

BCG vaccination should be considered only for children under 16 years of age with negative tuberculin reactions who:

- belong to groups with new infection rates of greater than 1 per cent per year;

- cannot be placed on isoniazid therapy but have uninterrupted exposure to people with active disease; or

- have continuous exposure to patients with organisms resistant to isoniazid and rifampicin.

\section{CHEMOPROPHYLAXIS}

- Unless specifically contraindicated, isoniazid chemoprophylaxis should be offered to all people at high risk of TB, including:

- tuberculin positive HIV infected people;

- tuberculin positive contacts of people with TB;
- tuberculin negative children under five years who are close contacts of people with TB. Prophylaxis should continue until a repeat test in 12 weeks is shown also to be negative;

- tuberculin positive people with medical risk factors for TB;

- tuberculin positive intravenous drug users;

- people who have recently converted tuberculin positive;

- people with positive tuberculin tests and chest $\mathrm{x}$-rays suggestive of inactive $\mathrm{TB}$;

- people in whom active disease is excluded and who have not completed treatment for TB;

- other tuberculin positive people under 35 years of age (including immigrants).

$\square \quad$ Preventive therapy should usually be with isoniazid for six to twelve months.

- Rifampicin treatment should be considered for people at high risk of severe forms of disease who are likely to be infected with isoniazid resistant organisms. People receiving chemoprophylaxis should be reviewed at least monthly for side-effects and to assess compliance.

\section{CASE FOLLOW-UP}

- People with infectious TB (pulmonary TB with acid fast bacilli seen on direct examination of a sputum smear) should be treated in a single hospital room until at least two weeks of anti-TB treatment has been taken.

- Treatment should be supervised by chest clinic staff for the duration of therapy, especially if there is any doubt about the patient's compliance.

口 Patients successfully treated for TB or infection need not be followed up routinely; they should be given a copy of their latest chest $x$-ray and advised about symptoms and to seek medical care immediately if symptoms suggestive of TB appear.

\section{EVALUATION}

- The value of screening should be assessed regularly. A cost-effectiveness analysis of the migrant screening program should be conducted and recommendations made about its usefulness.

The elimination of TB from NSW is an achievable goal. More emphasis is required in targeting appropriate screening efforts towards, and offering chemoprophylaxis to, those people most at risk of TB. The authors would welcome comments on this proposal through the Public Health Bulletin.

Jeremy McAnulty, Michael Levy, George Rubin Epidemiology and Health Services Evaluation Branch NSW Health Department

1. Centers for Disease Control. Recommendations of the Immunization Practices Advisory Committee (ACIP). Use of BCG vaccines in the control of tuberculosis: a joint statement by the ACIP and the Advisory Committee for Elimination of Tuberculosis. MMWR 1988; 37:663-675. 2. Centers for Disease Control. Update: Tuberculosis elimination United States. MMWR 1990; 39:153-156.

3. Reider HL, Cauthen GM, Comstock GW, Snider DE. Epidemiology 3. Reider HL, Cauthen GM, Comstock GW, Snider DE. E

of tuberculosis in the US. Epidemiol Rev 1989; 11:79-98.

4. Centers for Disease Control. Tuberculosis and human immunodeficiency virus infection: Recommendations of the Advisory Cammittee for the Elimination of Tuberculosis (ACET). MMWR 1989; $38: 237-250$.

5. National Health and Medical Research Council. Tuberculosis in Australia and New Zealand into the 1990s. 1989. Australian Government Publishing Service, Canberra.

6. Centers for Disease Control. A strategic plan for the elimination of tuberculosis in the United States. MMWR 1989; 38(suppl no s-3):1-24. 